\title{
RENDIMENTO GRAVIMÉTRICO EMTANINOS CONDENSADOS NAS CASCAS DE Anadenanthera peregrina EM DIFERENTES CLASSES DIAMÉTRICAS
}

\author{
Caroline Junqueira Sartori ${ }^{1 *}$, Fábio Akira Mori ${ }^{1}$, Mara Lúcia Agostini Valle ${ }^{1}$, Lourival Marin Mendes ${ }^{1}$, Thiago de \\ Paula Protásio ${ }^{1}$
}

*Autor para correspondência: krolsartori@hotmail.com

\begin{abstract}
RESUMO: O objetivo deste trabalho foi determinar o rendimento gravimétrico em taninos condensados nas cascas de Anadenanthera peregrina em diferentes classes diamétricas. Coletaram-se amostras de cinquenta e nove árvores de Anadenanthera peregrina, a 1,30 $\mathrm{m}$ do solo (Diâmetro a altura do peito - DAP), distribuídas em sete classes diamétricas. As cascas foram secas ao ar e trituradas em moinho de martelo. Foram realizadas amostragem composta para o preparo do extrato. A extração foi feita em água, na relação 15:1 (v/p), adicionados $3 \%$ de sulfito de sódio (v/v) em banho-maria a $70^{\circ} \mathrm{C}$ por 4 horas. O material foi filtrado com emprego de coador de pano fino e concentrado em chapa de aquecimento a aproximadamente $150 \mathrm{~g}$. Foi determinada a massa do extrato e retirados $10 \mathrm{~g}$ para a obtenção do teor de sólidos e $20 \mathrm{~g}$ para a para o índice de Stiasny. Os valores médios de teor de sólidos totais, índice de Stiasny, teor de tanino condensado e teor de compostos não tânicos foram de $11,34 \% ; 75,79 \% ; 12,76 \%$ e $4,07 \%$, respectivamente. O teor de sólidos, índice de Stiasny, teor de compostos não tânicos tiveram diferenças significativas entre as classes diamétricas. Já para a produção de taninos condensados, o parâmetro classe diamétrica não influenciou.
\end{abstract}

Palavras-chave: Angico-vermelho, índice de Stiasny, compostos fenólicos, adesivo natural.

\section{TANNINS GRAVIMETRIC YIELD CONDENSED IN Anadenanthera peregrina BARK IN DIFFERENT DIAMETER CLASSES}

\begin{abstract}
This work aimed to determine the gravimetric yield of condensed tannins in the Anadenanthera peregrina bark in different diameter classes. Fifty-nine trees samples were collected of Anadenanthera peregrina, at 1.30m of the ground (diameter at breast height - DBH), distributed in seven diameter classes. The barks were dried and crushed in mill of hammer. Composite sample was made to prepare the extract. The extraction was done using water in the ratio $15: 1$ ( $v / w)$, added 3\% sodium sulfite (w/w) in waterbath at $70^{\circ} \mathrm{C}$ for 4 hours. The material was filtered using fine cloth strainer and concentrated on a heating plate at approximately 150 $\mathrm{g}$. It was determined the extract mass and removed $10 \mathrm{~g}$ for obtaining solids content and $20 \mathrm{~g}$ for the Stiasny's index. The average values of total solids content, Stiasny's index, condensed tannin content and the compound content non-tannin were 11.34\%; 75.79\%; $12.76 \%$ and $4.07 \%$, respectively. The content of solids, Stiasny's index, compound content non-tannin show significant differences between diameter classes. For the condensed tannins production, the diameter class parameter there was no influence.
\end{abstract}

Keywords: Red-angico, Stiasny's index, phenolic compounds, natural adhesive.

\section{INTRODUÇÃO}

Entre as espécies produtoras de taninos se destacam as espécies de angico (Anadenanthera sp.), pertencentes à família Fabaceae, subfamília Mimosoideae. Diversas são as espécies de angico ocorrentes no Brasil, das quais se extrai, da casca, os taninos (AFONSO, 2008). Segundo o mesmo autor, a casca de angico é um dos principais produtos não madeireiros ocorrentes no Cerrado.

A utilização das cascas de angico-vermelho para extração de taninos é uma alternativa economicamente viável tendo em vista a ampla gama de utilizações possíveis dos taninos e por se tratar de uma espécie pioneira, de rápido crescimento, o que promove uma redução nos resíduos da indústria de transformação da madeira e agregação de um maior valor às cascas.

1 Universidade Federal de Lavras - Lavras, Minas Gerais, Brasil
Os taninos vegetais ou naturais são, por definição, substâncias que possuem a propriedade de se associar e de se combinar com proteínas e com certos polióis. Este comportamento é à base das propriedades tanantes que eles exercem sobre o colágeno da pele dos animais ao curso de sua transformação em couro (PIZZI, 1993). Os taninos ocorrem amplamente nos vegetais, porém, sua extração comercial é realizada através da casca e, ou, do cerne da madeira, locais onde são encontrados em maiores teores e também onde costumeiramente ocorrem os maiores problemas com injúrias e ataques de agentes xilófagos da madeira (TRUGILHO et al., 2003).

Os taninos são classificados, de acordo com sua origem biossintética, em dois grupos: a) os taninos hidrolisáveis, encontrados em dicotiledôneas lenhosas e herbáceas, que são caracterizadas por um núcleo (glicose)

Cerne, Lavras, v. 20, n. 2, p. 239-244, abr./jun. 2014 
esterificado com ácidos gálicos (galotaninos), ou ácidos elágicos (elagitaninos), formado a partir da rota metabólica do ácido chiquímico e b) os taninos condensados ou proantocianidinas, que ocorrem principalmente em gimnospermas e angiospermas e são polímeros da flavan3-ol e, ou, flavan-3,4-diol, derivados do metabolismo de fenilpropanóides (BRUNETON, 1991).

Além das funções biológicas desempenhadas, os taninos são utilizados no curtimento do couro, na indústria farmacêutica, alimentícia, produção de tintas, purificação de águas residuais e indústrias de adesivos naturais para madeira.

Adesivos à base de taninos são obtidos reagindose flavonóides poliméricos de origem natural, ou seja, taninos condensados, com um aldeído, normalmente o formaldeído, como agente ligante (PIZZI, 1994). Para a produção dos adesivos à base de taninos, são utilizados os taninos condensados, pois são quimicamente mais estáveis e economicamente mais viáveis. Esses taninos são constituídos de uma mistura de flavonóides polimerizados, de pesos moleculares variados, chamados genericamente de proantocianidinas (CARNEIRO et al., 2009). Os taninos condensados constituem mais de $95 \%$ da produção mundial de taninos comerciais (GUANGCHENG et al., 1991).

Já os taninos hidrolisáveis possuem certas propriedades indesejáveis, como baixa reatividade com formaldeído e baixo caráter nucleofílico. Estas características químicas, associadas à limitada produção mundial, impede que eles sejam efetivamente utilizados para a produção de adesivos fenólicos (PIZZI; MITTAL, 2003).

Diante do exposto, o objetivo desse trabalho foi determinar o rendimento gravimétrico em taninos condensados nas cascas de Anadenanthera peregrina, em diferentes classes diamétricas, de modo a analisar a viabilidade de utilização de indivíduos oriundos de desbaste na produção de adesivos naturais para madeira.

\section{MATERIAL E MÉTODOS}

\subsection{Caracterização da área de estudo e coleta do material}

O plantio de angico (Anadenanthera peregrina) pertence a uma área experimental do campus da Universidade Federal de Lavras (Lavras-MG). A cidade de Lavras localiza-se na região sul do Estado de Minas Gerais, à latitude de $21^{\circ} 14^{\prime}$ sul e longitude $45^{\circ} 00^{\prime}$ oeste, a uma altitude média de $900 \mathrm{~m}$. Segundo a classificação de Köppen, o clima é Cwa. A temperatura média anual do ar é de $19,4{ }^{\circ} \mathrm{C}$ e o total anual de precipitação pluvial de $1530 \mathrm{~mm}$ (BRASIL, 1992).

Coletaram-se cascas externa de Anadenanthera peregrina na altura de $1,30 \mathrm{~m}$ do solo (DAP) tomandose cuidado para não atingir o cambio vascular. A coleta foi realizada com facão, retirando-se amostras de aproximadamente $10 \mathrm{~cm}$ de comprimento por $3 \mathrm{~cm}$ de largura, de maneira intercalada ao longo do perímetro do fuste, de maneira a evitar o contorno total do mesmo. Foram coletadas, amostras de cinquenta e nove árvores, distribuídas em sete classes diamétricas, conforme pode

Tabela 1 - Características diamétricas do plantio de Anadenanthera peregrina e dos indivíduos utilizados na etapa experimental.

Table 1 - Diametric characteristics Anadenanthera peregrine plantation and of the individuals used in the experimental stage.

\begin{tabular}{ccccc}
\hline ICD $(\mathrm{cm})$ & $\begin{array}{c}\text { VCD } \\
(\mathrm{cm})\end{array}$ & $\begin{array}{c}\text { Diâmetro Médio } \\
\text { das Amostras } \\
(\mathrm{cm})\end{array}$ & $\begin{array}{c}\text { Altura Média das } \\
\text { Amostras }(\mathrm{m})\end{array}$ & $\begin{array}{c}\text { Número } \\
\text { de Árvores } \\
\text { Amostradas }\end{array}$ \\
\hline 5 a 9,99 & 7,5 & $7,15(11,90 \%)$ & $8,0(15,11 \%)$ & 14 \\
10 a 14,99 & 12,5 & $12,04(10,63 \%)$ & $10,94(17,76 \%)$ & 9 \\
15 a 19,99 & 17,5 & $16,54(3,58 \%)$ & $14,0(17,90 \%)$ & 8 \\
20 a 24,99 & 22,5 & $22,27(4,83 \%)$ & $14,61(16,40 \%)$ & 9 \\
25 a 29,99 & 27,5 & $27,23(4,90 \%)$ & $15,86(14,53 \%)$ & 7 \\
30 a 34,99 & 32,5 & $31.17(3,11 \%)$ & $17,0(15,22 \%)$ & 6 \\
35 a 39,99 & 37,5 & $37,25(2,62 \%)$ & $18,33(10,15 \%)$ & 6 \\
\hline
\end{tabular}

ICD: Intervalo das classes diamétricas; VCD: Valor central da classe diamétrica; Os valores em parênteses correspondem aos coeficientes de variação dentro das classes diamétricas

ser visto na Tabela 1. Os indivíduos amostrados não pertenciam à borda do plantio. O material foi coletado durante o mês de julho de 2011.

As cascas foram secas ao ar e moídas em moinho de martelo. Após a moagem, as amostras foram armazenadas em sacos de papel abertos e mantidas em sala de climatização com temperatura e umidade relativa do ar constantes $\left(20 \pm 2{ }^{\circ} \mathrm{C}\right.$ e $\left.60 \pm 5 \%\right)$, até massa constante. Posteriormente, foi determinada a umidade na base seca.

Cerne, Lavras, v. 20, n. 2, p. 239-244, abr./jun. 2014 


\subsection{Preparo dos extratos e quantificação de taninos}

Foram realizadas análises composta para cada classe, sendo a classe 1 analisada em um único extrato, e as demais classes em duplicata. Para a extração, foram colocados em béquer de $2000 \mathrm{~mL}$ o correspondente a $100 \mathrm{~g}$ de cascas moídas, $3 \mathrm{~g}$ de sulfito de sódio $\left(\mathrm{Na}_{2} \mathrm{SO}_{3}\right)$ e $1500 \mathrm{~mL}$ de água destilada, e levados em banho-maria à temperatura de $70^{\circ} \mathrm{C}$, durante quatro horas. Após a extração, o material foi filtrado com o emprego de coador de pano fino.

O filtrado foi concentrado por evaporação até um volume de aproximadamente $150 \mathrm{~mL}$, em chapa de aquecimento e capela de refluxo. A massa do extrato foi determinada e foram retiradas duas amostras de $10 \mathrm{~g}$ para a determinação do teor de sólidos e duas amostras de 20 g para determinação do índice de Stiasny. Para a determinação do teor de sólidos (\%), as amostras foram levadas à estufa, a uma temperatura de $103 \pm 2^{\circ} \mathrm{C}$ até massa constante, sendo obtido pela relação da massa seca com a massa úmida (inicial) da amostra, ao multiplicar por 100, conforme Equação 1:

$\mathrm{TST}(\%)=\mathrm{MS} / \mathrm{MU} \times 100$

em que:

TST é o teor de sólidos totais, em porcentagem;

MS é a massa da alíquota seca; e

MU é a massa inicial da alíquota.

O índice de Stiasny foi determinado empregandose o procedimento adotado por Mori (2000). A cada amostra de $20 \mathrm{~g}$ que foi retirada do extrato concentrado, adicionaram-se $10 \mathrm{~g}$ de água destilada, $4 \mathrm{ml}$ de formaldeído $(37 \% \mathrm{~m} / \mathrm{m})$ e $2 \mathrm{~mL}$ de $\mathrm{HCl} 10 \mathrm{~N}$. Essa mistura foi aquecida durante 35 minutos sob refluxo.

Após o término da reação, o extrato foi filtrado em filtro de vidro sinterizado de porosidade 2 e disposto em estufa à temperatura de $103 \pm 2^{\circ} \mathrm{C}$ até massa constante. Após obter a massa seca do precipitado, calculou-se o índice de Stiasny conforme Equação 2:

$\mathrm{IS}=\mathrm{M} 2 / \mathrm{M} 1 \times 100$

em que:

IS: Índice de Stiasny (\%);

M1: Massa total de sólidos em $20 \mathrm{~g}$ de extrato (g); e M2: Massa seca do precipitado tanino-formaldeído (g).
O rendimento em sólidos foi obtido pela multiplicação do teor de sólidos (g) e a massa de cada extrato. Para se obter o rendimento gravimétrico em taninos (\%), foi multiplicado o rendimento em sólidos pelo respectivo índice de Stiasny de cada extrato. O rendimento em componentes não tânicos foi obtido pela diferença entre o rendimento em sólidos e o rendimento em taninos.

\subsection{Análise estatística}

Foi realizado o Teste de Bartlett a 5\% para verificar a homogeneidade de variâncias e também o teste ShapiroWilk a 5\% para verificar a normalidade dos resíduos.

$\mathrm{Na}$ avaliação do experimento considerou-se o delineamento inteiramente casualizado tendo como fator de variação o valor central da classe. Os dados foram submetidos a análises de variância a 5\% de significância e quando verificada diferenças estatísticas significativas foi realizada a análise de regressão linear simples.

Foram realizadas análises de correlação linear de Pearson a 5\% de significância, entre índice de Stiasny e teor de compostos não tânicos.

\section{RESULTADOS E DISCUSSÃO}

Os valores médios de teor de sólidos totais (TST $\%$ ), índice de Stiasny (IS \%), teor de taninos condensados (TTC\%) e teor de compostos não tânicos (TNT \%) estão apresentados na Tabela 2.

Tabela 2 - Valores médios pelo método de Stiasny.

Table 2 - Mean values by the method of Stiasny.

\begin{tabular}{ccccc}
\hline VCD $(\mathrm{cm})$ & TST (\%) & IS (\%) & TTC (\%) & TNT (\%) \\
\hline 7,5 & 11,89 & 71,54 & 12,76 & 5,08 \\
12,5 & 11,23 & 71,61 & 12,07 & 4,77 \\
17,5 & 12,97 & 77,33 & 14,10 & 4,12 \\
22,5 & 10,75 & 77,86 & 12,55 & 3,56 \\
27,5 & 11,03 & 77,63 & 12,86 & 3,69 \\
32,5 & 10,38 & 78,78 & 12,26 & 3,30 \\
37,5 & 11,16 & 75,75 & 12,74 & 4,00 \\
\hline Média & 11,34 & 75,79 & 12,76 & 4,07 \\
CV (\%) & 7,53 & 3,98 & 5,12 & 15,85 \\
\hline
\end{tabular}

VCD: Valor central da classe diamétrica; TST: Teor de sólidos totais; IS: Índice de Stiasny; TTC: Teor de tanino condensado; TNT: Teor de compostos não tânicos; CV: Coeficiente de variação.

Cerne, Lavras, v. 20, n. 2, p. 239-244, abr./jun. 2014 
O teor de sólidos totais, que expressa a quantidade de extrativos totais presentes nas amostras analisadas, foi em média de $11,34 \%$. O índice de Stiasny, quantidade de substâncias do extrato total que reagiu com o formaldeído em meio ácido, foi em média de $75,79 \%$. O teor de taninos condensados, que indica a proporção de taninos presente nos materiais analisados, foi em média de $12,76 \%$. O teor médio de compostos não tânicos foi de $4,07 \%$. Para a produção de adesivos naturais para madeira são desejáveis maiores índices de Stiasny e menores teores de compostos não tânicos.

Trugilho et al. (1997), estudando os teores de taninos nas cascas de espécies do cerrado, encontraram valores médios de $10,62 \%$ e $18,51 \%$ em angico cangalha Peltophorum dubim (Spreng. Taule.) e angico vermelho Anadenanthera macrocarpa (Benth) respectivamente.

Carneiro (2006), ao empregar 3\% de sulfito de sódio, encontrou valores médios de teor de sólidos, índice de Stiasny, teor de taninos condensados e teor de compostos não tânicos de $28,75 \%, 82,2 \%, 22,77 \%$ e 5,98\% respectivamente para Anadenanthera peregrina. Esse maior desempenho pode ter sido obtido pelo fato da extração ter sido realizada em autoclave $\left(70^{\circ} \mathrm{C}\right)$ e granulometria padronizada entre 40 e $60 \mathrm{mesh}$, sendo partículas menores, promovendo, desta forma uma maior superfície de contato entre as partículas e o solvente de extração.

O efeito do valor central da classe diamétrica foi significativo para o teor de sólidos totais, índice de Stiasny e teor de compostos não tânicos, não havendo diferença significativa para teor de taninos condensados pelo teste $\mathrm{F}$ a 5\% de significância. $O$ teor de taninos condensado não foi influenciado pelo fator valor central da classe diamétrica, sendo seus valores iguais estatisticamente à medida que se aumenta o DAP (Tabela 2).

A regressão linear simples (relação) entre teor de sólidos totais e o valor central da classe diamétrica encontra-se na Figura 1. Verifica-se que teor de sólidos totais apresentou tendência de diminuição com o aumento do valor central da classe diamétrica, porém o modelo de regressão em nível de 5\% de significância foi não significativo. $\mathrm{O}$ modelo da regressão foi significativo em nível de $6 \%$ de significância, porém possui baixo coeficiente de determinação $\left(\mathrm{R}^{2}=0,274\right)$. $\mathrm{O}$ modelo de regressão linear entre teor de sólidos totais e valor central da classe diamétrica foi pouco significativo.

A regressão parabólica (relação) entre índice de Stiasny e o valor central da classe diamétrica encontra-se na
Figura 2. Verifica-se um aumento nos índices de Stiasny até aproximadamente a classe correspondente ao valor central de $22,5 \mathrm{~cm}$, tendendo a estabilização e posterior redução do índice de Stiasny com o aumento do valor central da classe diamétrica.

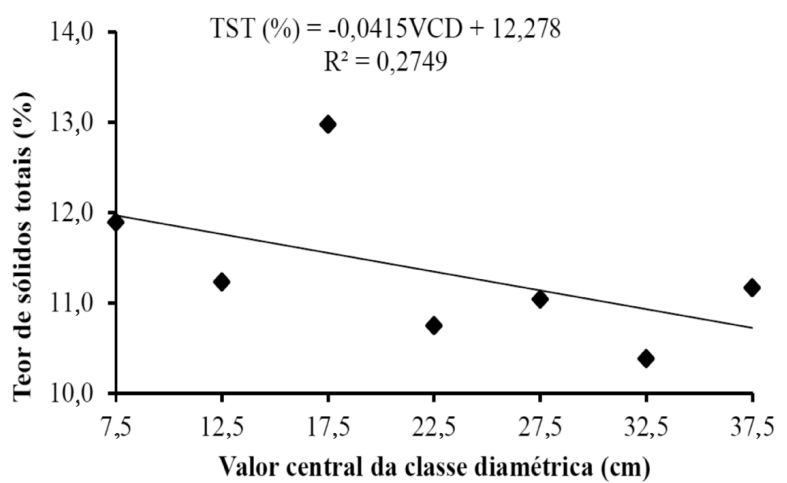

Figura 1 - Relação linear entre valor central da classe e teor de sólidos totais.

Figure 1 - Linear relationship between the central value class and total solids.

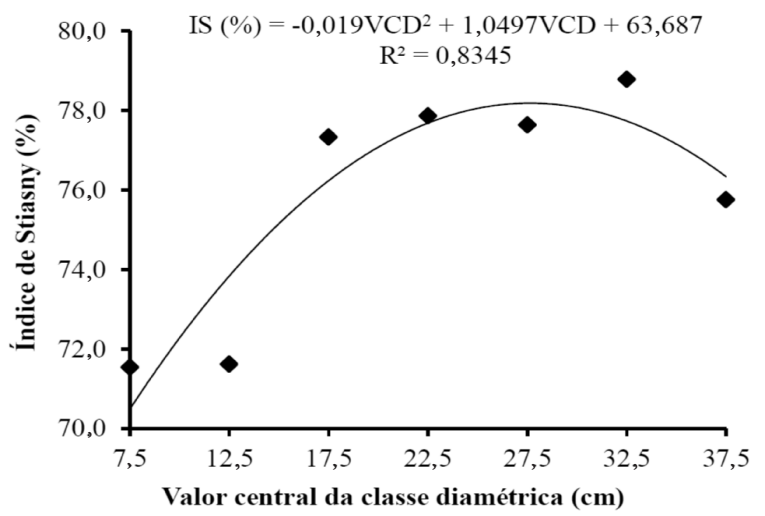

Figura 2 - Relação entre valor central da classe e índice de Stiasny.

Figure 2 - Relationship among the central value of the class and Stiasny"s index.

As árvores mais novas sintetizam compostos fenólicos mais simples, ou seja, os fenóis simples e ácidos fenólicos, e, à medida que aumenta sua idade aumenta a polimerização destes compostos formando os polímeros, que são os taninos e a lignina. Esta polimerização tende a se estabilizar e diminuir ao longo da idade da árvore.

Cerne, Lavras, v. 20, n. 2, p. 239-244, abr./jun. 2014 
Este comportamento dificulta a utilização de indivíduos provenientes de desbaste para produção de taninos para adesivos de madeira, pois nota-se que a produção nos indivíduos na classe de $7,5 \mathrm{~cm}$ foi em média de aproximadamente $71 \%$ e nos indivíduos da classe de diâmetro de $27,5 \mathrm{~cm}$ foi de aproximadamente $78 \%$. Indica-se para a produção de adesivos para madeira que o diâmetro dos indivíduos esteja na faixa de 22,5 até 32,5 $\mathrm{cm}$, pois a partir deste diâmetro o índice de Stiasny tendeu a diminuir, conforme Figura 2. A partir da derivada do modelo ajustado observa-se que o maior valor para o índice de Stiasny (aproximadamente 78\%) está compreendido para as árvores com diâmetro a altura do peito médio de $27,60 \mathrm{~cm}$.

A regressão parabólica (relação) entre teor de compostos não tânicos e o valor central da classe diamétrica encontra-se na Figura 3. Verifica-se uma redução nos teores de compostos não tânicos à medida que se aumenta o valor central da classe diamétrica. Este comportamento pode ser explicado da mesma maneira que para o índice de Stiasny. As árvores mais novas sintetizam compostos fenólicos mais simples, e a medida que aumenta sua idade aumenta a polimerização destes compostos formando os polímeros, que são os taninos, reduzindo assim a quantidade de compostos não tânicos no material. A partir da classe correspondente ao valor central de $22,5 \mathrm{~cm}$ a quantidade de compostos não tânicos tende a estabilizar e a partir da classe correspondente ao valor central de 32,5 tende a aumentar.

Para a produção de adesivos para madeira, recomenda-se a utilização de indivíduos com menores percentuais em compostos não tânicos, ou seja, aqueles que possuem DAP médio na faixa de $22,5 \mathrm{~cm}$ a $32,5 \mathrm{~cm}$, o que inviabiliza a utilização de indivíduos com diâmetros menores que $22,5 \mathrm{~cm}$. As classes que possuíram menores valores de compostos não tânicos foram às mesmas que apresentaram maiores índices de Stiasny, sendo estas as indicadas para a produção de adesivos de madeira. A partir da derivada do modelo encontra-se para qual valor de diâmetro é encontrado o menor teor de compostos não tânicos, que foi de $28,70 \mathrm{~cm}$.

A curva de regressão entre o teor de compostos não tânicos e o valor central da classe diamétrica teve comportamento inverso ao observado para o índice de Stiasny e o valor central da classe diamétrica. A correlação entre o índice de Stiasny e teor de compostos não tânicos foi de $-0,88$, conforme consta na Figura 4.

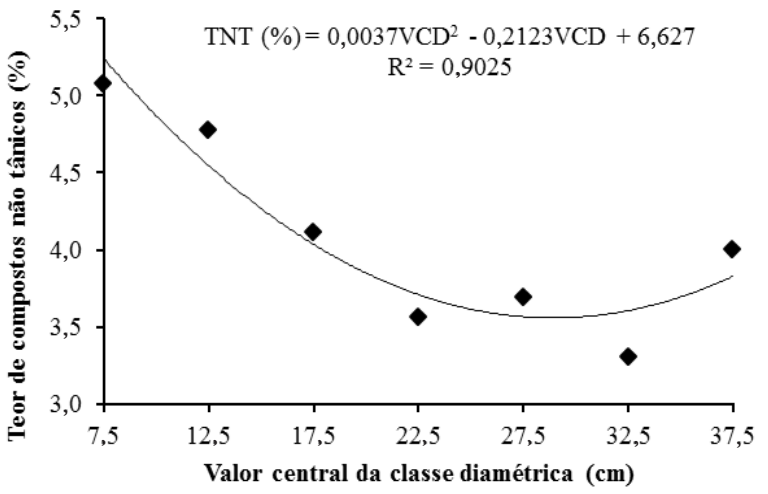

Figura 3 - Relação entre teor de compostos não tânicos e valor central da classe.

Figure 3 - Relationship between content of non-tannic compounds and the central value of the class.

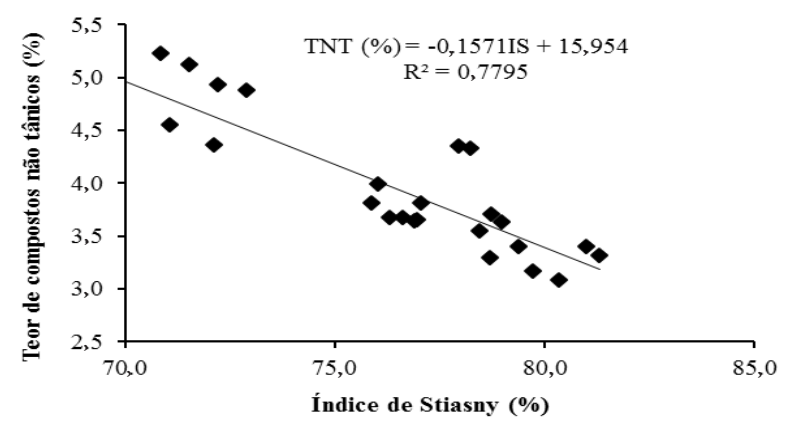

Figura 4 - Relação entre teor de compostos não tânicos e Índice de Stiasny.

Figure 4-Relationship between the content of non tannic compounds and Stiasny's Index.

\section{CONCLUSÕES}

O efeito do diâmetro a altura do peito não foi significativo para os teores de taninos condensados.

A produção de sólidos tendeu a diminuir com o aumento da classe diamétrica.

A regressão entre o índice de Stiasny e o valor central da classe diamétrica resultou em um modelo parabólico, sendo que o índice de Stiasny máximo foi encontrado em árvores correspondentes ao centro de classe de $27,5 \mathrm{~cm}$.

A regressão entre o teor de compostos não tânicos e o valor central da classe diamétrica resultou em um modelo

Cerne, Lavras, v. 20, n. 2, p. 239-244, abr./jun. 2014 
parabólico, sendo que o teor de compostos não tânicos mínimo foi encontrado em árvores correspondentes ao centro de classe de $27,5 \mathrm{~cm}$.

A utilização de indivíduos com diâmetros menores que $22,5 \mathrm{~cm}$ para a produção de adesivos não é recomendada, pois estes possuem menores índices de Stiasny e maiores teores de compostos não tânicos. Recomenda-se para a produção de adesivos naturais para madeira a utilização de indivíduos com diâmetros na faixa de 22,5 a $32,5 \mathrm{~cm}$.

\section{REFERÊNCIAS}

AFONSO, S. R. Análise sócio-econômica da produção de não-madeireiros no cerrado brasileiro e o caso da cooperativa de pequi em Japonvar, MG. 2008. 107 p. Dissertação (Mestrado em Ciências Florestais) Universidade de Brasília, Brasília, 2008.

BRASIL. Ministério da Agricultura e Reforma Agrária. Secretaria Nacional de Irrigação. Departamento Nacional de Meteorologia. Normais climatológicas: 1961-1990. Brasília, 1992. 84 p.

BRUNETON, J. Elementos de fitoquimica e de farmacognosia. Zaragoza: Acribia, 1991. 594 p.

CARNEIRO, A. C. O. Efeito da hidrólise ácida e sulfitação de taninos de Eucalyptus grandis w. hill ex maiden e Anadenanthera peregrina speg., nas propriedades dos adesivos. 2006. $182 \mathrm{f}$. Tese (Doutorado em Ciência Florestal) - Universidade Federal de Viçosa, Viçosa, 2006.

CARNEIRO, A. C. O.; VITAL, B. R.; FREDERICO, P. G. U.; CARVALHO, A. M. M. L.; VIDAURRE, G. B. Propriedades de chapas de aglomerado fabricadas com adesivo tânico de angico-vermelho (Anadenanthera peregrina) e uréia-formaldeído. Revista Árvore, Viçosa, v. 33, n. 3, p. 521-531, maio/jun. 2009.

GUANGCHENG, Z.; YUNLU, L; YAZAKI, Y. Extractive yields, Stiasny values and polyflavonoid contents in barks from six acacia species in Australia. Australian Forestry. v.54, p.154 -156, 1991.

MORI, F. A. Caracterização parcial dos taninos da casca e dos adesivos produzidos de três espécies de Eucaliptos. 2000. 73 f. Tese (Doutorado em Ciência Florestal) - Universidade Federal de Viçosa, Viçosa, 2000.

Cerne, Lavras, v. 20, n. 2, p. 239-244, abr./jun. 2014
PIZZI, A. Advanced wood adhesives technology. New York: M. Dekker, 1994. 289 p.

PIZZI, A. Tanin-based adhesives. In: Wood adhesives: chemistry and techonology. New York: M. Dekker, 1993. p. 177-246.

PIZZI, A.; MITTAL, K. L. Handbook of adhesive technology. 2nd ed. New York: M. Dekker, 2003. 720 p.

TRUGILHO, P. F.; CAIXETA, R. P.; LIMA, J. T.; MENDES, L. M. Avaliação do conteúdo em taninos condensados de algumas espécies típicas do cerrado mineiro. Cerne, Lavras, v. 3, n. 1, p. 1-13, 1997.

TRUGILHO, P. F.; MORI, F. A.; LIMA, J. T.; CARDOSO, D. P. Determinação do teor de taninos na casca de Eucalyptus spp. Cerne, Lavras, v. 9, n. 2, p. 246-254, 2003.

Recebido: 10 de abril de 2012; aceito: 04 de setembro de 2013. 